\title{
Sliding mode controller design for wood drying process
}

\author{
Zheng Zhou ${ }^{1,2} \cdot$ Keqi Wang ${ }^{1}$
}

Received: 25 June 2017 / Published online: 11 April 2018

(C) The Author(s) 2018

\begin{abstract}
This paper deals with the design of sliding mode controllers for the wood drying process. Prediction models based on a nonlinear autoregressive model with exogenous inputs of dry-bulb temperature and equilibrium moisture content during drying were built to design the controllers. A comparative study was performed in order to determine the controller presenting the better performance in practical application. The compared strategies were sliding mode control based on exponent approach law and variable rate approach law. The second controller was introduced to optimize the controller while reducing the chattering phenomenon in comparison with the exponent approach law. To demonstrate the effectiveness of the proposed control strategies, simulation and experimental results were presented.
\end{abstract}

\section{Introduction}

Kiln drying of wood has been a common way for reducing moisture content (MC) down to a level at which the wood is suitable for normal service, like furniture, joinery and construction materials (Konopka et al. 2015). Wood drying automatic control systems play a crucial role in the timber manufacture. Wood, as an inhomogeneous material, has a complicated structure, and the quality of wood products is greatly dependent upon the dry-bulb temperature and equilibrium moisture content (EMC) during the drying process (Naveros et al. 2015; Chaiyo and Rattanadecho 2013). Previous studies (e.g., Situmorang and Situmorang 2015) demonstrate that the control variables of wood drying process usually include dry-bulb temperature

Keqi Wang

zdhwkq@163.com

1 Mechanical and Electrical Engineering College, Northeast Forestry University, Harbin, China

2 Electrical and Information College, Heilongjiang Bayi Agricultural University, Daqing, China 
and EMC as they are the two main factors influencing the drying performance. Hence, it is important to maintain the desired dry-bulb temperature and EMC in order to have a satisfactory performance in autonomous mode for wood drying.

Prediction models based on nonlinear autoregressive models with exogenous inputs (ARX) have been widely shown in the literature (Ben Abdelwahed et al. 2017; $\mathrm{Hu}$ et al. 2012; Filipovic 2015; Beyhan and Alci 2010). Given the need for accurate predictions, a nonlinear ARX model for wood drying process is built to design the controllers in the study. Nowadays, the required level of autonomy for wood drying operations has not been fully developed yet. The need to reduce drying time and simultaneously improve the drying quality has become an issue for any of the systems previously mentioned (Shibata and Hirohashi 2013). Sliding mode control (SMC) that is appealing to practical applications has shown a strong antiinterference ability. A SMC controller is a robust controller that can be applied to both linear and nonlinear systems (Amini et al. 2017). Previous studies (e.g., Edelberg et al. 2013; Amini et al. 2014; Shahbakhti et al. 2015) have indicated the capabilities of SMC dealing with implementation and model uncertainties. SMC was initially used in the continuous system due to the damage caused by the chattering in discontinuous system. To overcome this deficiency, a large amount of improvements to reduce the chatter have been developed. In Korkut and Guller (2007), Sun et al. (2011), Hsu and Chang (2016) and Tang et al. (2013), intelligent, adaptive and fractional enhancements for the chattering reduction have been investigated. Likewise, the methods based on exponent approach law have been proposed in Wang et al. (2016) and Hou and Zhang (2016). The researches have proven that exponent approach control law preserves the main advantages of SMC while improving the control accuracy. SMC based on exponent approach law is used to design the wood drying controller in this study. SMC based on variable rate approach law is also introduced to optimize the exponent approach control law.

This paper is aimed at the SMC controller design for wood drying process. This work is applied to poplar (Populus ussuriensis Kom), a typical wood species from Northeast China, which has an important potential market in Chinese timber manufacturing industry. The first contribution of this study is to build the prediction model using actual field measured data from the small-size wood drying kiln. The second contribution is to design the wood drying controllers based on SMC method. This paper is organized as follows: in "Sliding mode controller design" section, a SMC controller based on the exponent approach law is introduced. An improved control strategy based on variable rate approach law is also presented. "Simulation results" section is the numerical simulation to verify effectiveness of the proposed methods. Furthermore, experimental results are presented in "Experimental results" section. Finally, some conclusions are drawn in "Conclusion" section. 


\section{Sliding mode controller design}

\section{Nonlinear ARX model for wood drying prediction}

Wood moisture content mainly depends on the dry-bulb temperature and EMC during wood drying, which are controlled by the three valves, i.e., heater, sprayer and damper. Nonlinear ARX model uses the historical data of the three valves to predict the future data of the dry-bulb temperature and the EMC. In the experiment, the operative mode of the three valves was taken as inputs, and the dry-bulb temperature and the EMC measured by sensors installed in the kiln were regarded as outputs of the prediction model.

Dry-bulb temperature and EMC prediction model based on nonlinear ARX algorithm is given in Eq. (1),

$$
y(t)=\sum_{i=1}^{n} a_{i} f(y(t-i))+\sum_{j=1}^{m} b_{j} h(u(t-j))+v(t),
$$

where $u(t)$ and $y(t)$ are the input and the output of the model, $f(y(t))$ and $h(u(t))$ are the nonlinearized static functions, $f(t)$ is a nonlinear function that maps the output of the linear block to the system output, $h(t)$ is a nonlinear function transforming input data $u(t), v(t)$ is the disturbance of the model, and $a_{i}$ and $b_{j}$ represent the autoregressive parameters.

Figures 1 and 2 give the simulation results based on the nonlinear ARX model for wood drying. Wellness of fit indicates how accurately the models can estimate the dry-bulb temperature and the EMC for wood drying process.

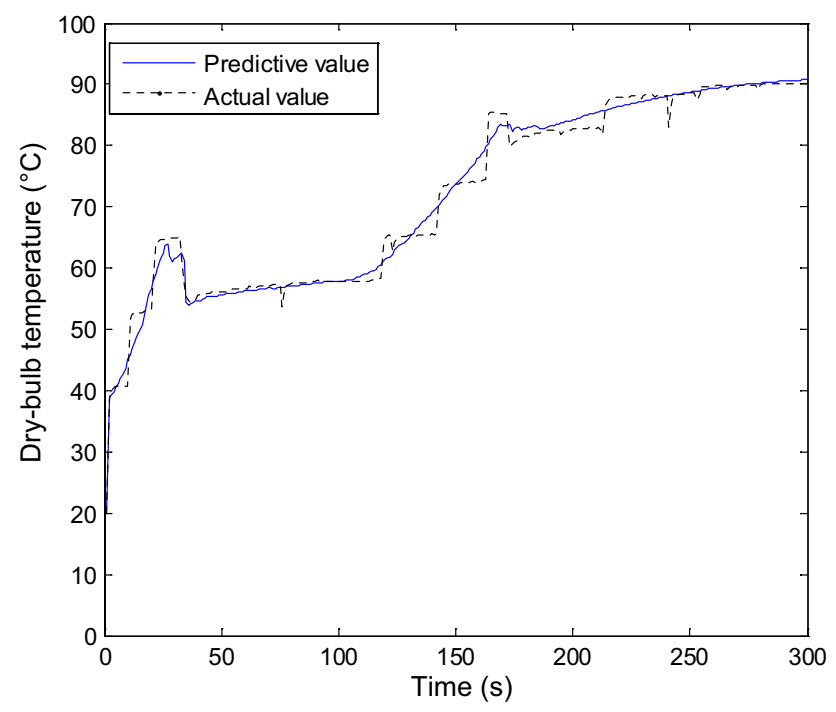

Fig. 1 Simulation results of dry-bulb temperature for wood drying process 


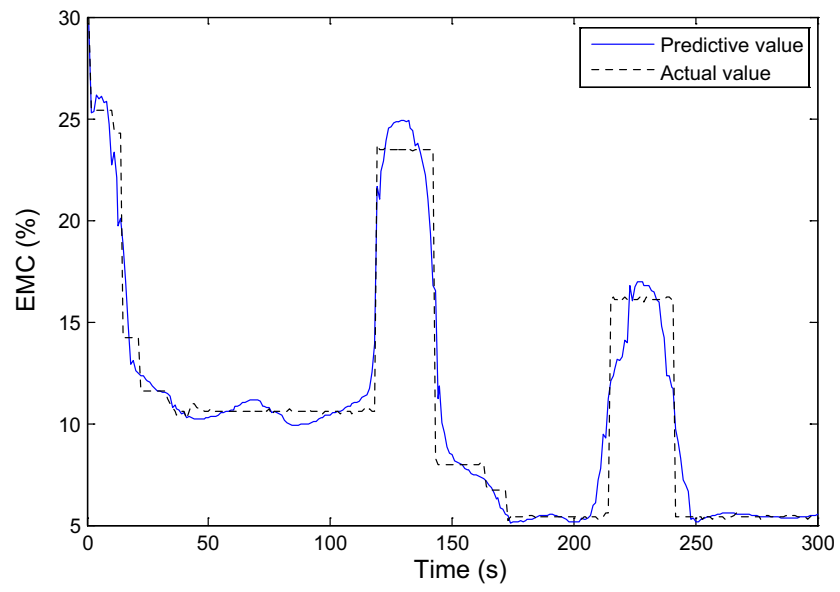

Fig. 2 Simulation results of EMC for wood drying process

\section{Sliding mode controller design}

In this section, two strategies based on SMC theory are presented for wood drying control. The mathematic model given by Eq. (1) is linearized to reduce the complexity and improve the efficiency of the control system. The linearized mathematical model describing the dry-bulb temperature and the EMC for wood drying is given as

$$
x(k+1)=A x(k)+B u(k),
$$

where $x(k)=\left[x_{1}(k), x_{2}(k)\right]$.

$r(k)$ is position function, and its change rate is $\mathrm{d} r(k)$. Let $R=[r(k) ; \mathrm{d} r(k)]$, $R 1=[r(k+1) ; \mathrm{d} r(k+1)]$. Linear extrapolation method is employed to predict $r(k+1)$ and $\mathrm{d} r(k+1)$,

$$
r(k+1)=2 r(k)-r(k-1), \mathrm{d} r(k+1)=2 \mathrm{~d} r(k)-\mathrm{d} r(k-1) .
$$

The sliding mode surface $s$ is designed as

$$
s(k)=C_{\mathrm{e}} E=C_{\mathrm{e}}(R(k)-x(k)),
$$

where $C_{\mathrm{e}}=[c 1]$, and $c>0$. According to Eqs. (2) and (4),

$$
s(k+1)=C_{\mathrm{e}}\left(R(k+1)-C_{\mathrm{e}} A x(k)-C_{\mathrm{e}} B u(k) .\right.
$$

The objective of the controller is to enforce the sliding mode into the surface $s=0$. The proposed control law is given by the following equation,

$$
u(k)=(C e B)^{-1}(C e R(k+1)-C e A x(k)-s(k+1)) .
$$

The approach law based on exponent is given by

$$
s(k+1)=s(k)+T(-e q \operatorname{sgn}(s(k))-q s(k)) .
$$

Substitute Eq. (7) into Eq. (8), the control law based on exponent is obtained 


$$
u(k)=(C e B)^{-1}(C e R(k+1)-C e A x(k)-s(k)-d s(k)),
$$

where $d s(k)=-e q T \operatorname{sgn}(s(k))-q T s(k)$.

$q, e q$ and $c$ are the three parameters in SMC based on exponent approach law. $q$ is the main factor influencing the dynamic transition. The switching function $\mathrm{Ce}$ that affects the stability and response time is determined by the value of sliding surface parameter $c$. Gain parameter of the sign function $e q$ is employed to avoid perturbation and external disturbance. There is a disadvantage of exponent control law in that the phase locus of the system always chatters near the origin point. The chatter may stimulate unstable system dynamics, degrading the overall control performance in real-time implementations. Variable rate approach law is introduced to improve the system performance, which ensures that the phase locus reaches the origin point.

The discrete function of variable speed reaching law is given as

$$
s(k+1)-s(k)=-\varepsilon T\|x(k)\| \operatorname{sgn}(s(k)) .
$$

Substitute Eq. (9) into Eq. (6), the variable rate approach control law is obtained,

$$
u(k)=(C e B)^{-1}(C e R(k+1)-C e A x(k)-s(k)-d s(k)),
$$

where $d s(k)=-\varepsilon T\|x(k)\| \operatorname{sgn}(s(k))$.

\section{Simulation results}

In this section, the simulation results are presented to show the validity and performance of the proposed sliding mode controllers. Exponent approach law (EA-SMC) and variable rate approach law (VRA-SMC) employed to design the controllers are given by Eqs. (8) and (10). The proposed control methods were implemented by a computer code written in MATLAB ${ }^{\circledR}$. To verify the effectiveness of the sliding mode control algorithms, the initial dry-bulb temperature and EMC were set at

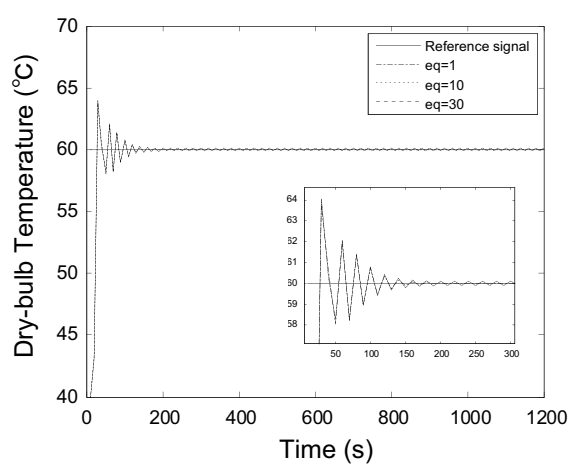

(a) EA-SMC

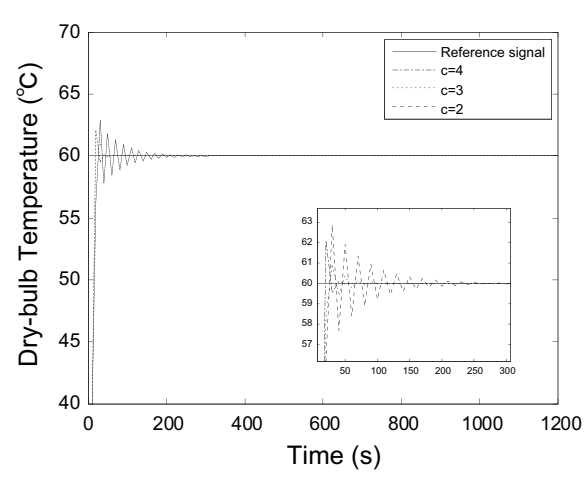

(b) VRA-SMC

Fig. 3 Simulation response of dry-bulb temperature. a EA-SMC. b VRA-SMC 


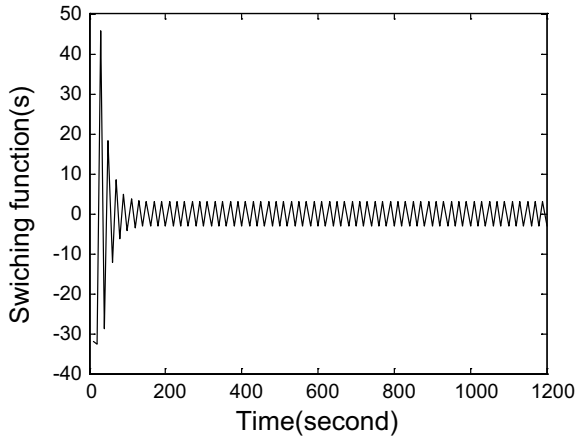

(a) EA-SMC

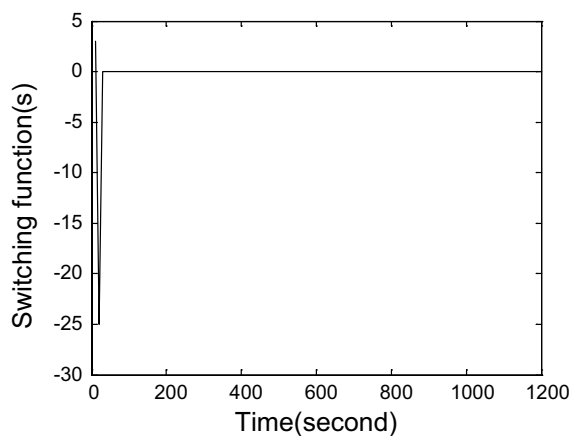

(b) VRA-SMC

Fig. 4 Switching function of dry-bulb temperature. a EA-SMC. b VRA-SMC

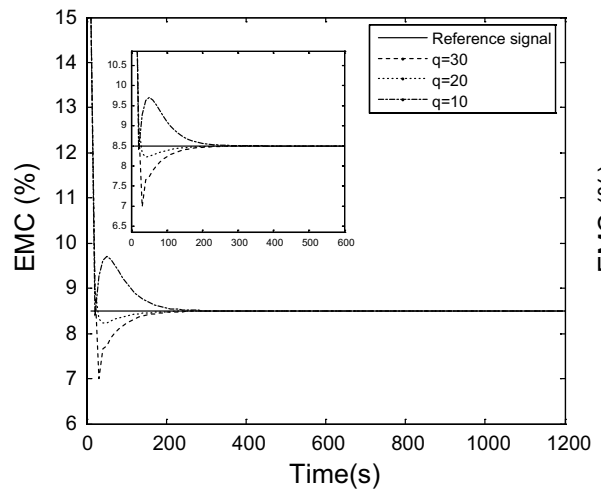

(a) EA-SMC

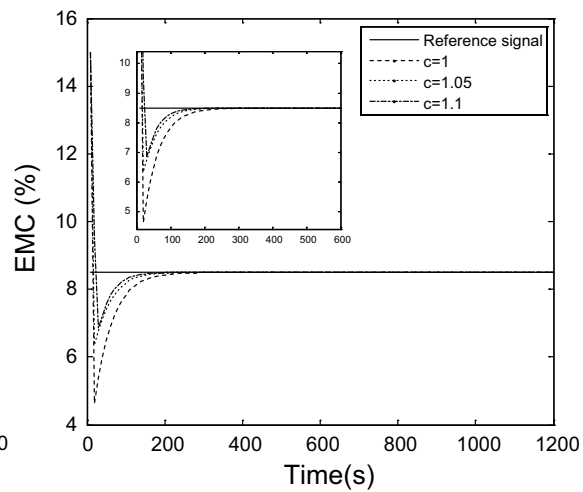

(b) VRA-SMC

Fig. 5 Simulation response of EMC. a EA-SMC. b VRA-SMC

$40{ }^{\circ} \mathrm{C}$ and $15 \%$, and the reference dry-bulb temperature and EMC were set at $60{ }^{\circ} \mathrm{C}$ and $8.5 \%$, respectively. The simulation results for the two sliding mode control laws proposed are shown in Figs. 3, 4 and 5.

The control performance indicates that the two controllers efficiently follow the reference signal. Figure 3 shows the simulation results of the dry-bulb temperature controllers. The parameters and control performance of dry-bulb temperature controllers are shown in Table 1. Figure 3 a shows the simulation results of EA-SMC with $e q=1, e q=10$ and $e q=30$ when $c=3, q=40$. The response time is $33 \mathrm{~s}$, and the overshoot is $6.23 \%$ with $\mathrm{eq}=1$. The control outputs chatter near the reference signal with $e q$ increases $(e q=10$ and $e q=30)$. Figure $1 \mathrm{~b}$ shows the simulation results of VRA-SMC with $c=4, c=3$ and $c=2$ when $e q=1$. It can be observed that the response time is shortest with $\mathrm{c}=2$ and longest with $\mathrm{c}=4$, the overshoot is smallest with $c=3$ and largest with $c=1$. 
Table 1 Comparison of the control indices of the dry-bulb temperature controllers

\begin{tabular}{llll}
\hline Controller & Control parameters & Control indices & \\
\cline { 3 - 4 } EA-SMC & $e q(c=3, q=40)$ & Overshoot $(\%)$ & Stable time $(\mathrm{s})$ \\
\hline & 1 & 6.23 & 33 \\
& 10 & - & - \\
\hline VRA-SMC & 30 & - & - \\
\hline & $c(e q=1)$ & & 66 \\
& 4 & 4.77 & 24 \\
& 3 & 1.56 & 10 \\
\hline
\end{tabular}

Figure 4 is the switching function of dry-bulb temperature using exponent approach law $(c=3, q=40, e q=10)$ and variable rate approach law $(c=3, e q=1)$. With regard to the switching function chattering, compared with EA-SMC controller, there is a substantial chattering reduction using VRA-SMC controller.

Figure 5 depicts the control performance of the two controllers for EMC. The parameters and control performance of EMC controllers are shown in Table 2. Figure 5a shows the simulation response obtained from EA-SMC with $q=30, q=20$ and $q=10$ when $c=1$ and $e q=1$. It can be appreciated that the controller presents the shortest response time and the smallest overshoot with $q=20$. The system response has a largest overshoot with $q=30$. The response time with $\mathrm{q}=30$ and $\mathrm{q}=10$ is the same. Figure $5 \mathrm{~b}$ shows the control signal obtained from VRA-SMC with $c=1, c=1.05$ and $c=1.1$ when $e q=1$. It can be seen that the controller presents the shortest response time with $c=1.1$ and the longest time with $c=1$, the overshoot is largest with $c=1$ and smallest with $c=1.1$.

Table 2 Comparison of the control indices of the EMC controllers

\begin{tabular}{llll}
\hline Controller & Control parameters & Control indices & \\
\cline { 3 - 4 } EA-SMC & $q(c=1, e q=1)$ & Overshoot $(\%)$ & Stable time $(\mathrm{s})$ \\
\hline 30 & 20 & 17.73 & 51 \\
& 10 & 3.24 & 49 \\
& $c(e q=1)$ & 14.13 & 51 \\
\hline VRA-SMC & 1 & & 54 \\
\hline & 1.05 & 45.43 & 43 \\
& 1.1 & 25.24 & 37 \\
\hline
\end{tabular}




\section{Experimental results}

In this section, the results obtained applying the proposed control techniques to wood drying process are presented. A small-size wood drying kiln developed at Northeast Forestry University, China, was used to evaluate the control performance. The size of the wood sample was $600 \mathrm{~mm} \times 140 \mathrm{~mm} \times 50 \mathrm{~mm}$, and the average initial moisture content and initial density were above $60 \%$ and $0.390 \mathrm{~g} /$ $\mathrm{cm}^{3}$, respectively. The sensors were installed in the drying kiln to collect the dry-bulb temperature during drying, in order to compare the drying performance when using the EA-SMC and VRA-SMC controllers. As shown in Fig. 6, the correlation of the set dry-bulb temperature and actual dry-bulb temperature indicates the effectiveness of the two control techniques. Improvement in tracking the reference value of the VRA-SMC controller can be seen compared with the EASMC controller.

Furthermore, the drying quality and visible flaws of each control strategy are presented in Tables 3 and 4. The drying process via EA-SMC started with a initial MC of $60 \%$ and finished with a final MC of $6.78 \%$. The MSE of MC was $0.78 \%$. The average residual stress was $0.24 \%$. Surface checks, internal checks and end checks were found in 3,2 and 5\% of the total samples, respectively. Color changing defects were not found. Cell collapse occurred in $2 \%$ of the samples. The

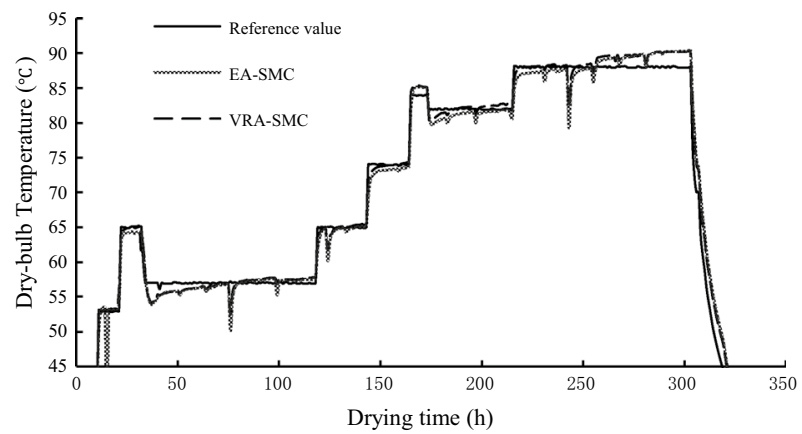

Fig. 6 Comparison of dry-bulb temperature curve during drying

Table 3 Index of experimental results

\begin{tabular}{lll}
\hline Drying result index & Algorithm \\
\cline { 2 - 3 } & EA-SMC & VRA-SMC \\
\hline Drying time (h) & 347 & 339 \\
Energy consumption (kw·h) & 2047 & 1980 \\
Vapor flow (kg) & 17,274 & 17,205 \\
Final MC (\%) & 6.78 & 7.08 \\
MSE of MC (\%) & 0.78 & 1.28 \\
Average residual stress (\%) & 0.24 & 1.75 \\
\hline
\end{tabular}


Table 4 Visiable flaw

\begin{tabular}{lll}
\hline Flaw & Algorithm & \\
\hline & EA-SMC & VRA-SMC \\
Surface checks & $3 \%$ & $2 \%$ \\
Internal checks & $2 \%$ & $2 \%$ \\
End checks & $5 \%$ & $4 \%$ \\
Color changing & None & None \\
Collapse & $2 \%$ & $2 \%$ \\
\hline
\end{tabular}

checks and collapse in $90 \%$ of the samples were acceptable. According to these indexes, the first-class standard was achieved.

The drying process used VRA-SMC, starting with the same initial MC as EASMC and finishing with a final MC of $7.08 \%$. The drying time of VRA-SMC was $8 \mathrm{~h}$ shorter than EA-SMC. With regard to the drying energy consumption, it translates into a $67 \mathrm{kw} \mathrm{h}$ and $69 \mathrm{~kg}$ reduction with VAR-SMC in electricity and water consumption. The MSE of MC and the average residual stress were 1.28 and $1.75 \%$, respectively. Surface checks, internal checks and end checks were found in 2, 2 and $4 \%$ of the total samples. Color changing was not found in the experimental samples. Cell collapse occurred in $2 \%$ of the samples. The checks and collapse in $90 \%$ of the samples were acceptable. According to these indexes, the first-class standard was achieved with VRA-SMC.

The indexes, which were final MC, MSE of MC, average residual stress and the flaws, used to evaluate the drying quality indicated that both the samples achieved the first-class standard using the two control strategies. Visible flaws of the wood samples indicated that the performance of the two controllers was approximate. However, as far as energy and water consumption were concerned, VRA-SMC had a better performance with regard to saving energy consumption and improving the profitability of the operation.

\section{Conclusion}

To achieve the level of autonomy for wood drying operation, sliding mode controllers based on the prediction model built using actual field measured data for wood drying process are investigated in this paper. The study on two sliding mode controllers based on exponent approach law and variable rate approach law for wood drying control is presented. The simulation results exhibit the effectiveness of the proposed control laws for the dry-bulb temperature and EMC control during wood drying process. The comparative study allows to conclude that the parameters, $c, q$ and $e q$, have a significant impact on the response time and the overshoot of the system. The chattering phenomenon of EA-SMC is obvious with $e q$ increases. The improvement can be seen for the chattering reduction in VRA-SMC in comparison with the simulation response obtained from EA-SMC. In field practice, the final MC, MSE of MC, average residual stress and flaws of 
wood samples dried via the two control strategies both achieved the first-class quality standard, and VRA-SMC had a better performance with regard to energy and water saving.

Open Access This article is distributed under the terms of the Creative Commons Attribution 4.0 International License (http://creativecommons.org/licenses/by/4.0/), which permits unrestricted use, distribution, and reproduction in any medium, provided you give appropriate credit to the original author(s) and the source, provide a link to the Creative Commons license, and indicate if changes were made.

\section{References}

Amini MR, Shahbakhti M, Ghaffari A (2014) A novel singular perturbation technique for modelbased control of cold start hydrocarbon emission. SAE Int J Eng 7(3):1290-1301

Amini MR, Shahbakhti M, Pan S, Hedrick JK (2017) Bridging the gap between designed and implemented controllers via adaptive robust discrete sliding mode control. Control Eng Pract 59:1-15

Ben Abdelwahed I, Mbarek A, Boutrara K (2017) Adaptive MPC based on MIMO ARX-Laguerre model. ISA Trans 67:330-347

Beyhan S, Alci M (2010) Fuzzy functions based ARX model and new fuzzy basis function models for nonlinear system identification. Appl Soft Comput 10(2):439-444

Chaiyo K, Rattanadecho P (2013) Numerical analysis of heat-mass transport and pressure build-up in 1D unsaturated porous medium subjected to a combined microwave and vacuum system. Drying Technol 31(6):684-697

Edelberg K, Shahbakhti M, Hedrick JK (2013) Incorporation of implementation imprecision in automotive control design. In: American Control Conference June 17-19: 2854-2859

Filipovic VZ (2015) Recursive identification of multivariable ARX models in the presence of a priori information: robustness and regularization. Sig Process 116:68-77

Hou H, Zhang Q (2016) Finite-time synchronization for second-order nonlinear multi-agent system via pinning exponent sliding mode control. ISA Trans 65:96-108

Hsu C-F, Chang C-W (2016) Intelligent dynamic sliding-mode neural control using recurrent perturbation fuzzy neural networks. Neurocomputing 173:734-743

Hu B, Zhao Z, Liang J (2012) Multi-loop nonlinear internal model controller design under nonlinear dynamic PLS framework using ARX-neural network model. J Process Control 22(1):207-217

Konopka A, Baranski J, Hurakova T, Klement I (2015) The influence of high temperature wood drying conditions using air-steam mixture on its properties. For Wood Technol 90:107-114

Korkut S, Guller B (2007) Comparison of two drying schedules for European hophornbeam (Ostrya carpinifolia Scop.) lumber. Drying Technol 25(12):1977-1984

NaverosMesa I, Ghiaus C, Ruiz DP, Castano Castano S (2015) Physical parameters identification of walls using ARX models obtained by deduction. Energy Build 108:317-329

Shahbakhti M, Reza Amini M, Li J, Asami S, Hedrick JK (2015) Early model-based design and verification of automotive control system software implementations. J Dyn Syst Meas Contr 137(2):021006

Shibata H, Hirohashi Y (2013) Effect of segment scale in a pore network of porous materials on drying periods. Drying Technol 31(7):743-751

Situmorang Z, Situmorang JA (2015) Intelligent fuzzy controller for a solar energy wood dry kiln process. Int Conf Technol Inf Manag Eng Environ IEEE 2015:152-157

Sun T, Pei H, Pan Y, Zhou H, Zhang C (2011) Neural network-based sliding mode adaptive control for robot manipulators. Neurocomputing 74(14-15):2377-2384

Tang Y, Zhang X, Zhang D, Zhao G, Gun X (2013) Fractional order sliding mode controller design for antilock braking systems. Neurocomputing 111:122-130

Wang Z, Zhou Y, Lee G (2016) The sliding mode control about ASR of vehicle with four independently driven in-wheel motors based on the exponent approach law. Energy Procedia 88:827-832 